SCIREA Journal of Clinical Medicine

ISSN: 2706-8870

http://www.scirea.org/journal/CM

August 15, 2021

SCIREA

Volume 6, Issue 5, October 2021

\title{
When sickle cell trait meets high-altitude
}

\author{
Sun Yong Lee ${ }^{1, *}$ and Amandeep S Gill ${ }^{2}$ \\ ${ }^{1}$ San Joaquin General Hospital, Internal medicine'; sunyong.lee55@gmail.com \\ ${ }^{2}$ San Joaquin General Hospital, Hematology \& Oncology 2; asgill@sjgh.org \\ * Correspondence: sunyong.lee55@gmail.com; Tel.:+1-209-468-6624
}

\begin{abstract}
:
Approximately 300 milion people worldwide have sickle cell trait and it is considered as a benign carrier state with relative protection to malaria. Despite of occasional reports, sickle cell trait can be associated with significant life-threatening coditions under certain situations such as hypoxia, dehydration, and strenuous physical exercise. Splenic infarct is one of the clinical complication from increasing red blood cell sickling and polymerization under low oxygen tension at high altitudes. It is crucial to be aware of the possible clinical complications with sickle cell trait and management to prevent morbidity and mortality.
\end{abstract}

Keywords: Sickle cell trait; splenic infarct; high altitude

\section{The case presentation:}

A 21-year-old Caucasian male with no known medical history presented to ED in Lake Tahoe with complaining of severe abdominal pain. The patient reported that abdominal pain was on the left upper quadrant, 10 out of 10 strength, associated with fever for the past 3 days. 
However, it was not associated with nausea or vomiting and no sick contact noted. He was traveling Lake Tahoe (6,237 feet) from Stockton, CA. He underwent blood test which showed sickle cell trait with hemoglobin A 57.3\%, hemoglobin S 38.7\% in hemoglobin electrophoresis. Total bilirubin was elevated at $2.7 \mathrm{mg} / \mathrm{dL}$ with direct bilirubin at $0.8 \mathrm{mg} / \mathrm{dL}$. CT abdomen/pelvis reported mild splenomegaly $13.5 \mathrm{~cm}$, splenic vein thrombosis, concerning for small area of splenic infarct (Figure A). He was prescribed rivaroxaban for anticoagulation in ED and was discharged. The patient was referred to our hematology/oncology clinic and underwent complete hypercoagulable state work-up including protein $\mathrm{C}$, protein $\mathrm{S}$, Antithrombin III, antiphospholipid panel, factor V Leyden mutation, myeloproliferative disorder including JAK 2 mutation, MPL mutation, CAL R mutation, CSF 3R mutation which were all negative. Repeat CT of the chest/abdomen in 2 months was normal without thrombosis (Figure B). Therefore, rivaroxaban was stopped as splenic infarct was due to sickle cell trait with exposure to high altitude in Lake Tahoe and repeat $\mathrm{CT}$ scan did not show any other abnormalities. The patient's abdominal pain resolved and was recommended to get adequately hydrated if he plans to go to high altitude area. Most cases of splenic infarction in sickle cell trait including acute splenic syndrome, can be successfully managed with hydration, oxygen, rest, analgesia, and other supportive measures without surgical intervention

Figure A. CT scan on ED presentation with splenic infarct

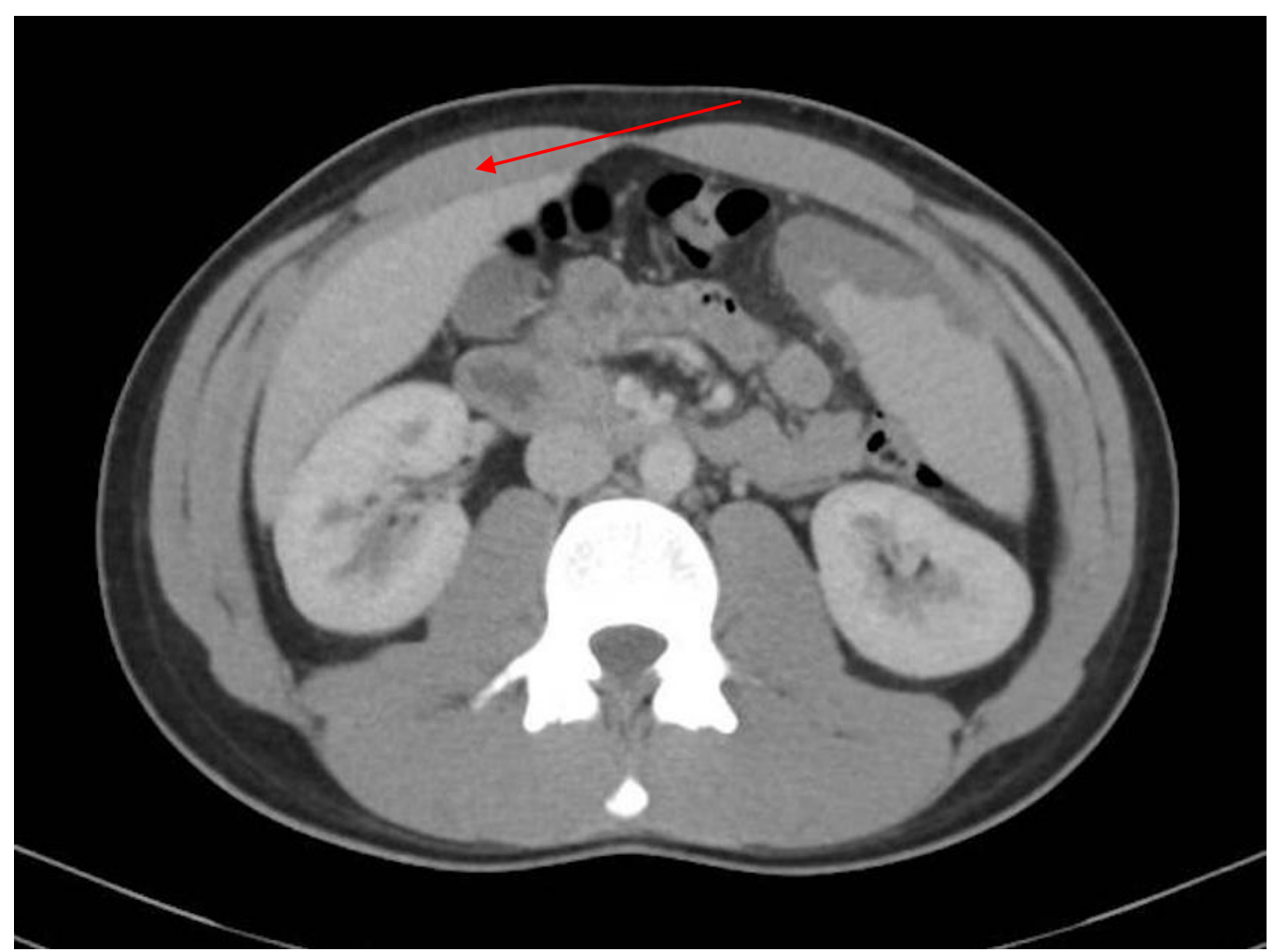


Figure B. Follow-up CT scan in 2 months later with resolved splenic infarct

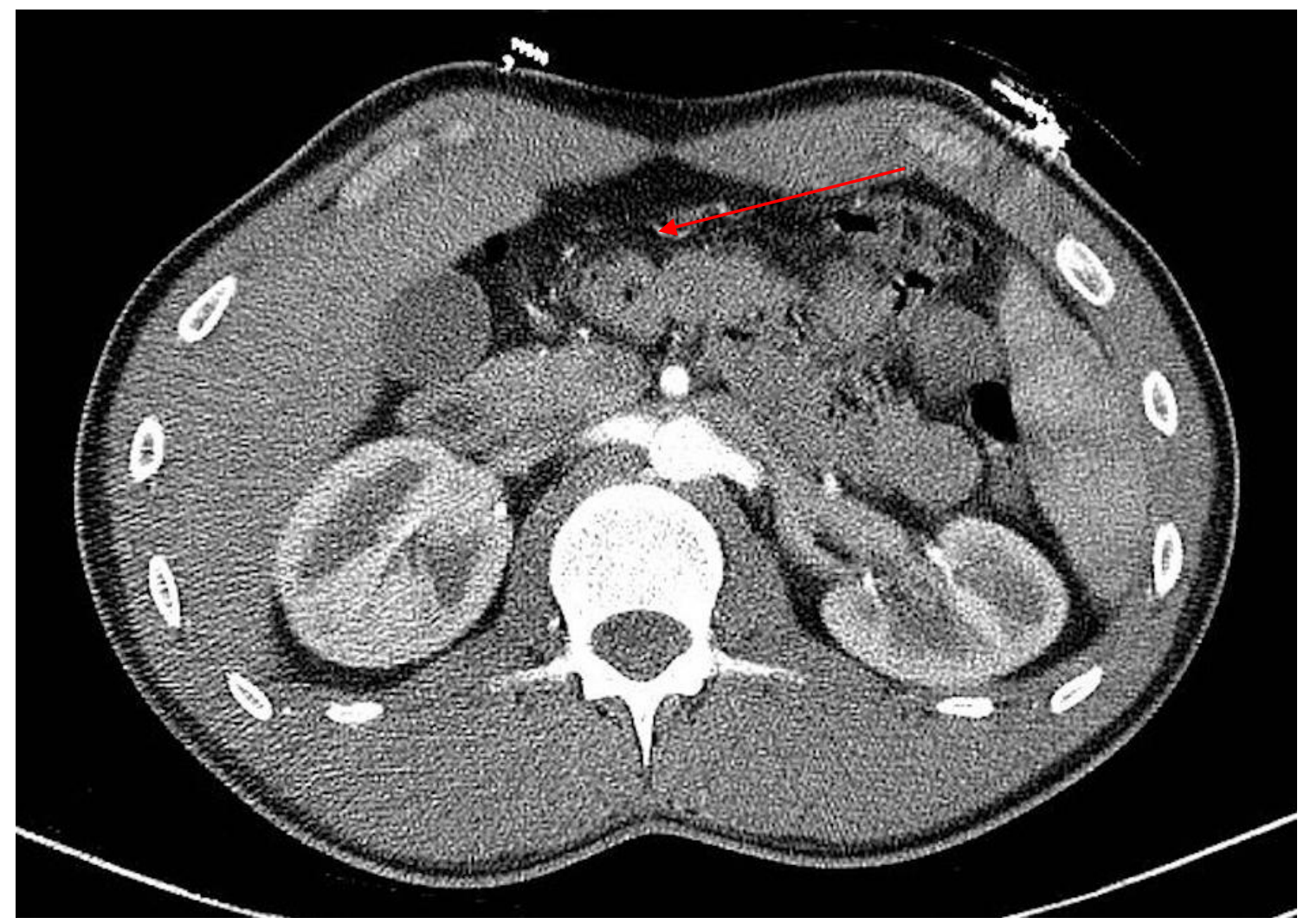

\section{References}

[1] Complications associated with sickle cell trait: a brief narrative review, Geoffrey Tsaras, Amma Owusu-Ansah, Freda Owusua Boateng, Yaw Amoateng-Adjepong, Am J Med. 2009 Jun; 122(6): 507-12. doi: 10.1016/j.amjmed.2008.12.020. Epub 2009 Apr 24.

[2] Acute splenic infarction in a hiker with previously unrecognised sickle cell trait, Monica Gupta, S S Lehl, Kamal Singh, Ram Singh, BMJ Case Rep. 2013 Apr 29; 2013: bcr2013008931. doi: 10.1136/bcr-2013-008931. 